\title{
Ocular hypertension and systemic responses to the water-drinking test
}

\author{
A. F. WINDER, A. A. SIDDIQUI, AND H. C. DONOVAN \\ From the Department of Pathology and Computer Section, Institute of Ophthalmology and Moorfields Eye \\ Hospital, London
}

SUMMARY Osmolal, electrolyte, and other responses in peripheral blood were recorded after water loading of patients with ocular hypertension and chronic simple glaucoma, and with a small group of younger normal subjects. The osmolal responses of the ocular hypertensive group were significantly greater than for the glaucoma group, and further differences in plasma potassium concentration were also found. Drinking habits may exert a significant influence in ocular hypertension. The relative contributions to the diagnostic category of the variables recorded was evaluated by discriminant analysis, and the application of this retrospective approach to the assessment of new patients is discussed.

Assorted versions of the water-drinking test have been widely used in the diagnosis and prognostic assessment of disorders of intraocular pressure, but the test is generally held to be of limited value for the individual case, particularly if assessment is based solely on pressure responses (Roth, 1974). The value of the test may possibly be improved by increased or body-weight-dependent water loads (Galin et al., 1965; Spaeth, 1967), repeat assessment (Drance, 1958; Frankelson, 1974), rejection of tests in which a fall of serum osmolality is not achieved (Drance, 1958), coadministration of additional agents such as pitressin (El-Shewy, 1973) and combination with outflow studies (Vucicevic et al., 1975), although this latter combination does not seem to be useful with ocular hypertension (Kronfeld, 1975). The aspects of ocular dynamics which are stressed by a water load have not yet been unequivocally defined, but the test response clearly has mechanical aspects, although further influences must also be involved (Iverson and Brown, 1967; Spaeth, 1967). The changes of intraocular pressure and serum osmolality are then likely to be causally related, although in timing and magnitude the agreement may not be close (Campbell et al., 1955; Galin et al., 1965; Spaeth, 1967; Spaeth and Vacharat, 1972). With ocular hypertension the pressure responses to a water load are similar to those with chronic simple glaucoma (Roth, 1974), perhaps reflecting the influence of the initial tension

Address for reprints: Dr A. F. Winder, Department of Pathology, Institute of Ophthalmology, Judd Street, London WC1H 9QS on the degree of response, and Galin et al. (1963 found that the osmolal changes in peripheral blood taken after a fasting water load were not obviously different with groups of glaucoma, suspect glaucoma, and other ophthalmological patients.

In view of the growing interest in systemic aspects of disorders of intraocular pressure we have further studied the responses to a water load in ocular hypertension and chronic simple glaucoma, with additional limited studies on normal subjects. Intraocular pressures were not recorded during the tests, for which assessment derives from osmolal, electrolyte, and other data obtained by the analysis of peripheral blood samples. Water loads were taken without fasting, partly as this difference may not be critical (Roth, 1974) and partly because it seemed possible that personal drinking habits might exert a significant influence on the intraocular pressure in ocular hypertension because of an increased or prolonged osmolal response.

We wished to determine whether the peripheral blood responses to water loading differ significantly between the groups tested, and we have made use of discriminant analysis to identify and evaluate, via the differential weighting involved, those factors for which the greatest differences arise and which therefore could be aetiological pointers in this group of disorders.

\section{Patients and methods}

\section{DIAGNOSTIC CRITERIA}

These were reported previously (Winder et al., 1974), i.e., ocular hypertension $(\mathrm{OH})$ : an intraocular 
pressure of $21 \mathrm{mmHg}$ or above in a patient having open angles, and no other ocular pathology. Chronic simple glaucoma (CSG): an intraocular pressure of $22 \mathrm{mmHg}$ or above in a patient having open angles, pathological cupping of the optic disc, and a glaucomatous field defect.

Normal subjects: volunteers without known ocular abnormality but on whom no form of ophthalmological assessment was performed.

\section{PATIENTS}

These were recruited at the Glaucoma Unit of Moorfields Eye Hospital, High Holborn, London, WC1. Studies were performed on 21 patients with ocular hypertension-14 males of average age 60.7 years and 7 females of average age 66.4 years (overall range 51 to 78 years), and 10 patients with chronic simple glaucoma -7 males of average age 56 years and 3 females of average age 54.3 years (overall range 32 to 77 years). The normal group comprised 7 males of average age $37 \cdot 3$ years and 3 females of average age $51 \cdot 3$ years (overall range 23 to 65 years), i.e., a lower age range than the above groups of patients.

\section{WATER-DRINKING TEST}

Tests were performed at 10 a.m. without prior fasting. After an initial venepuncture subjects drank 1 litre of tap water at room temperature in less than 5 minutes, and further venepunctures were taken at 20, 40, and 60 minutes after drinking began. Patients sat quietly during the test without smoking and were not currently taking acetazolamide. Blood samples of about $2 \mathrm{ml}$ were collected by venepuncture into heparinised tubes, for which the amount of anticoagulant had been shown to exert a negligible influence on sample osmolality.

\section{ASSESSMENT}

The initial 20, 40, and 60 minute plasma samples obtained by centrifugation were analysed as follows: osmolality was determined in duplicate on $0.25-\mathrm{ml}$ samples by a freezing point method with the Advance model 3L osmometer; sodium and potassium were determined on plasma dilutions by automated flame photometry; and cholesterol was determined by an automated colorimetric method. Continuous quality control assessment was applied to all these techniques. Thirteen variables were therefore directly recorded for each subject in these tests, plus 2 derived variables-peak drop in osmolality, and integrated osmolal depression, the osmolal sum drop. This latter was calculated from the recorded osmolal data on an area basis as shown in Fig. 1, a correction being made for any slight variation in sample collection time where necessary, the units effectively

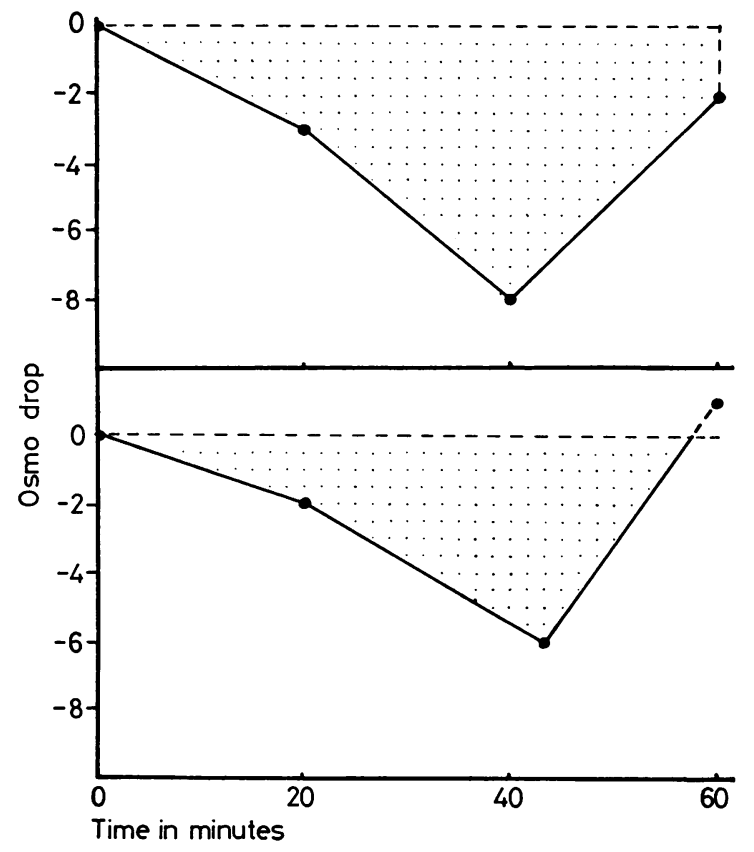

Fig. 1 Calculation of the osmolal sum drop. This is represented by the shaded area, start osmolality being recorded as 0 and variation above this level being discounted as in the lower diagram

being milliosmolal minutes experienced up to 60 minutes after water loading. Linearity between readings was necessarily assumed in these calculations, and excursions above the initial osmolal value were ignored, with manual graphical calculation of the appropriate osmolal sum drop. The group results for peak drop and osmolal sum drop shown in Table 1 are followed by repeat results recalculated after exclusion of occasional patients for whom a very low osmolal response to water loading was seen.

\section{STATISTICS}

All data were analysed by the Student's $t$ test and by discriminant analysis (SPSS programme). The component variables recorded were differentially weighted in either a stepwise manner-not necessarily using all variables, depending on performance -or a continuous, that is, all-in manner, to produce discriminant functions categorising all members of each subject group without overlap where possible. The necessary weighting involved reflects the relative importance of each variable in establishing the discriminant function, and if the group differences taken into account are valid, the relative importance of the factors assessed in the production of an abnormal response may also be inferred. 
Table 1 Water load responses for patients with chronic simple glaucoma, ocular hypertension and normal subjects, and the $P$ values obtained by intergroup comparison. Where subject numbers vary, the appropriate totals are shown in parentheses. (Osmo, Sod, and Pot represent plasma osmolality, and sodium and potassium levels at various times)

\begin{tabular}{|c|c|c|c|c|c|c|c|c|}
\hline \multirow{2}{*}{$\begin{array}{l}\text { Index } \\
\text { Subjects }\end{array}$} & \multicolumn{2}{|l|}{$C S G$} & \multirow[t]{2}{*}{$-P-$} & \multicolumn{2}{|l|}{$O H$} & \multirow[t]{2}{*}{$-P-$} & \multicolumn{2}{|l|}{ Normal } \\
\hline & & 10 & & & 21 & & & 10 \\
\hline Age & & $55 \cdot 5$ & & & $62 \cdot 6$ & & & $41 \cdot 5$ \\
\hline Osmo 0 & $278 \cdot 28 \pm$ & 7.91 & $0 \cdot 133$ & $282 \cdot 53 \pm$ & $6 \cdot 79$ & $0 \cdot 320$ & $277 \cdot 14 \pm$ & $4 \cdot 71$ \\
\hline Osmo 20 & $-3 \cdot 74 \pm$ & $4 \cdot 10$ & 0.066 & $-6 \cdot 19 \pm$ & $2 \cdot 91$ & 0.475 & $-5 \cdot 35 \pm$ & $3 \cdot 21$ \\
\hline Osmo 40 & $-5 \cdot 14 \pm$ & $2 \cdot 98$ & 0.028 & $-8 \cdot 60 \pm$ & $4 \cdot 23$ & 0.504 & $-7 \cdot 39 \pm$ & $5 \cdot 43$ \\
\hline Osmo 60 & $-2 \cdot 92 \pm$ & $2 \cdot 44$ & 0.016 & $-6 \cdot 61 \pm$ & $4 \cdot 24$ & 0.788 & $-6 \cdot 13 \pm$ & $5 \cdot 44$ \\
\hline Osmo Sum Drop & \multicolumn{2}{|c|}{$208.9 \pm 130 \cdot 8$} & 0.015 & \multicolumn{2}{|c|}{$357 \cdot 1 \pm 156 \cdot 5$} & $0 \cdot 449$ & \multicolumn{2}{|c|}{$309 \cdot 9 \pm 167 \cdot 8$ (10) } \\
\hline Osmo Sum Drop & \multicolumn{2}{|c|}{$228.8 \pm 121.6$} & 0.014 & \multicolumn{2}{|c|}{$373 \cdot 0 \pm 142 \cdot 2$} & - & \multicolumn{2}{|c|}{$339 \cdot 3 \pm 148.4$} \\
\hline Peak Drop & $6 \cdot 52 \pm$ & $3 \cdot 24(10)$ & 0.046 & $9 \cdot 39 \pm$ & $3 \cdot 71(21)$ & - & $9 \cdot 36 \pm$ & $5.47(10)$ \\
\hline Peak Drop & $7 \cdot 13 \pm$ & $2 \cdot 75 \quad(9)$ & 0.052 & $9 \cdot 76 \pm$ & $3 \cdot 39(20)$ & - & $10 \cdot 11 \pm$ & $5 \cdot 22 \quad(9)$ \\
\hline Subjects & & 9 & & & 17 & & & 10 \\
\hline Sod 0 & $139 \cdot 2 \pm$ & 8.03 & 0.859 & $139.8 \pm$ & $8 \cdot 20$ & 0.730 & $140 \cdot 8 \pm$ & $4 \cdot 16$ \\
\hline Sod 20 & $-2 \cdot 33 \pm$ & 3.57 & 0.654 & $-1 \cdot 77 \pm$ & $2 \cdot 73$ & 0.820 & $-2 \cdot 00 \pm$ & $2 \cdot 26$ \\
\hline Sod 40 & $-2 \cdot 89 \pm$ & $3 \cdot 18$ & 0.764 & $-3 \cdot 35 \pm$ & 3.95 & 0.670 & $-2 \cdot 70 \pm$ & 3.53 \\
\hline Sod 60 & $-1 \cdot 67 \pm$ & 5.07 & 0.459 & $-2 \cdot 76 \pm$ & $2 \cdot 44$ & 0.895 & $-2 \cdot 50 \pm$ & $7 \cdot 66$ \\
\hline Pot 0 & $4 \cdot 21 \pm$ & 0.59 & 0.382 & $4 \cdot 38 \pm$ & 0.38 & 0.008 & $3 \cdot 94 \pm$ & $0 \cdot 37$ \\
\hline Pot 20 & $-0 \cdot 067 \pm$ & 0.384 & 0.911 & $-0.053 \pm$ & 0.24 & 0.857 & $-0.07 \pm$ & 0.24 \\
\hline Pot 40 & $-0.067 \pm$ & 0.485 & 0.257 & $-0 \cdot 16 \pm$ & 0.46 & 0.283 & $-0.03 \pm$ & 0.37 \\
\hline Pot 60 & $-0.044 \pm$ & 0.472 & 0.430 & $-0 \cdot 13 \pm$ & 0.55 & 0.687 & $-0.05 \pm$ & 0.35 \\
\hline Subjects & & 9 & & & 19 & & & 10 \\
\hline Chol. 0 & $5 \cdot 30 \pm$ & 0.96 & 0.098 & $6.02 \pm$ & 1.06 & 0.863 & $5.92 \pm$ & 1.92 \\
\hline
\end{tabular}

\section{Results}

Data recorded for some patients were incomplete: the overall results and $P$ values obtained from Student's $t$ test are shown in Table 1, together with the number of patients involved in each case.

(A) CSG GROUP $v$. NORMAL GROUP

Considering individual variables no significant differences between the water load responses were found. Discriminant analysis: Both stepwise and direct approaches were applied as shown in Table 2. The function derived from the stepwise approach utilised 6 variables and correctly classified 16 of 19 subjects. When all variables and the direct approach were used, a function correctly classifying all subjects was derived.

(B) OH GROUP $v$. NORMAL GROUP

When individual variables were considered and age was excluded, significant differences were found in initial osmolality and initial plasma potassium concentration (Table 1). Discriminant analysis: A stepwise approach correctly classified 13 of $17 \mathrm{OH}$ and 8 of 10 normal subjects, i.e., 21 of 27 subjects

Table 2 CSG group v. normal group: discriminant analysis, by stepwise and direct procedures, listing the most useful variables and their standardised discriminant function coefficients

\begin{tabular}{|c|c|c|c|}
\hline \multicolumn{2}{|c|}{ Stepwise method } & \multicolumn{2}{|c|}{ Direct method } \\
\hline Variable & coeff. & Variable & Coeff. \\
\hline Pot 60 & $-1 \cdot 24819$ & Osmo sum & $-1 \cdot 85615$ \\
\hline Pot 0 & $-1 \cdot 21440$ & Pot 20 & $-1 \cdot 71312$ \\
\hline \multirow[t]{3}{*}{ Osmo sum } & 0.69521 & Pot 0 & $-1 \cdot 17217$ \\
\hline & & Osmo 20 & $-1 \cdot 11884$ \\
\hline & & Peak drop & $1 \cdot 05440$ \\
\hline
\end{tabular}


using only 3 variables. With the direct approach using all variables all 10 normal and 15 of $17 \mathrm{OH}$, i.e., 25 of 27 subjects, were correctly classified. The most important components are shown in Table 3.

(C) OH GROUP $v$. NORMAL GROUP $v$. CSG GROUP

All comparisons involving the normal group-and particularly this one-are speculative in view of the intergroup age differences involved. Applying discriminant analysis, functions were obtained with each approach correctly classifying 29 of 36 subjects -stepwise ( 7 variables) 13 of $17 \mathrm{OH}, 7$ of 9 CSG, and 9 of 10 normal; and direct 13 of $17 \mathrm{OH}, 8$ of 9 CSG, and 8 of 10 normal. The most useful information is shown in Table 4.

\section{(D) CSG GROUP $v$. OH GROUP}

When individual variables were considered, significant differences $(P<0.05)$ were found in the osmolality drop after 40 and 60 minutes $(20, P=$ 0.066), the peak drop and the osmolal sum drop (Table 1). The results for peak drop and osmolal drop sum shown in Table 1 are followed by repeat results recalculated after exclusion of occasional patients for whom a very low osmolal response to water loading was seen. Discriminant analysis:

Table 3 OH group v. normal group: discriminant analysis by stepwise and direct methods, listing the most useful variables and their standardised discriminant function coefficients

\begin{tabular}{llll}
\hline \multicolumn{2}{l}{ Stepwise method } & \multicolumn{2}{l}{ Direct method } \\
Variable & Coeff. & Variable & Coeff. \\
\hline Osmo 0 & 0.66908 & Osmo 40 & -1.38249 \\
Pot 0 & 0.59313 & Peak drop & -1.15868 \\
Sod 40 & -0.32550 & Pot 40 & -0.87030 \\
& & Osmo sum & 0.73593 \\
& & Osmo 0 & 0.71528 \\
\hline
\end{tabular}

Table 4 OH group v. normal group v. CSG group. Discriminant analysis by stepwise and direct methods, listing the most useful variables and their standardised discriminant function coefficients

\begin{tabular}{llll}
\hline \multicolumn{2}{l}{ Stepwise method } & \multicolumn{2}{l}{ Direct method } \\
Variable & Coeff. & Variable & Coeff. \\
\hline Pot 20 & -0.75994 & Osmo 40 & -1.91621 \\
Pot 40 & -0.71833 & Peak drop & -1.71488 \\
Osmo 0 & -0.60852 & Osmo 0 & -0.71367 \\
Pot 1 & -0.43258 & Pot 1 & 0.62205 \\
\hline
\end{tabular}

Table 5 CSG group v. OH group: discriminant analysis by stepwise and direct methods, listing the most useful variables and their standardised discriminant function coefficients

\begin{tabular}{lcll}
\hline \multicolumn{2}{l}{ Stepwise method } & \multicolumn{2}{l}{ Direct method } \\
Variable & Coeff. & Variable & Coeff. \\
\hline Osmo sum & 3.56106 & Osmo sum & -3.01671 \\
Osmo 40 & 2.28548 & Osmo 40 & -2.40828 \\
Osmo 20 & 1.42918 & Osmo 20 & -1.35276 \\
Osmo 60 & 1.26775 & Osmo 60 & -1.12483 \\
Sod 40 & 0.62422 & Osmo 0 & 0.66152 \\
Osmo 0 & -0.59068 & Peak drop & -0.55102 \\
\hline
\end{tabular}

Table 6 Osmolal water load response data for glaucoma, glaucoma suspect, and normal subjects, calculated from the results of Galin et al. (1963)

\begin{tabular}{lcccc}
\hline Index & Glaucoma & Suspect & Normal \\
\hline Subjects & 27 & 27 & 25 \\
Age & not stated & not stated & not stated \\
Osmo 0 & $287.6 \pm 3.26$ & $288.0 \pm 3.19$ & $289.1 \pm 2.91$ \\
Osmo sum & $391.9 \pm 131.5$ & $318.0 \pm 110.3$ & $352.9 \pm 115.4$ \\
Peak drop & $10.8 \pm 3.08$ & $8.82 \pm 2.29$ & $10.1 \pm 3.11$ \\
\hline
\end{tabular}

Functions were derived using both stepwise (10 variables) and direct approaches which correctly assigned all $17 \mathrm{OH}$ plus 8 of 9 CSG patients. The most relevant information is shown in Table 5 . The stepwise approach gave marginally better discrimination and the osmolal characteristics of the responses were clearly the most significant factors involved. Further analysis of the time course of the osmolal response indicated that a slightly higher proportion of $\mathrm{OH} v$. CSG subjects peaked at 40 minutes, with a reduced proportion of $\mathrm{OH} v$. CSG patients peaking before 40 minutes. There was no specific abnormality in the data of the CSG patient who fell in the $\mathrm{OH}$ group with both approaches, and this patient will be followed up clinically with interest.

(E) RECALCULATION OF PREVIOUS DATA Galin et al. (1963) presented osmolal responses for groups of normal subjects, glaucoma suspects, and those with established open-angle glaucoma, and we have reworked these data to compare initial osmolality, peak drop, and osmolal sum response as above, with results shown in Table 6. Intergroup differences were not significant in any respect, and we did not proceed to discriminant analysis. 


\section{Discussion}

In these experiments comparisons involving the normal group are speculative in view of its size and the age differences involved, but even so group allocation using discriminant analysis-which for retrospective allocation clearly involves a deal of circular argument-is surprisingly successful, and the differences involved are not necessarily simply age-related artefacts. The persistent group differences involving plasma potassium levels are particularly interesting, and we have since found that Spaeth (1967) and Iverson and Brown (1967) commented on the erratic responses of plasma potassium after water loading in studies of glaucoma suspects and patients with open-angle glaucoma. This phenomenon may be an important aetiological pointer and from contemporary studies adrenocortical and/or renal associations are obvious possibilities.

Direct comparison between the groups with ocular hypertension and chronic simple glaucoma indicates that the osmolal response of the former was significantly increased both in terms of peak drop and overall integrated hypotonic response up to 1 hour. Peak drop was also marginally later in the ocular hypertensive group, and plasma cholesterol values were increased, although not significantly so ( $\mathrm{P}<0 \cdot 1$ only) on this sample ( $c f$. Winder et al., 1974). Detectable osmolar responses to water loading continue long after 60 minutes (Spaeth, 1967; Iverson and Brown, 1967), but the substantial challenge is over by that time. Systemic differences in the water-load response profiles are therefore present, and drinking habits may indeed be important in terms of the ocular osmolar stress involved. This result also implies that local influences carry more weight in the lesion of chronic simple glaucoma.

Are these differences real? One reason for presenting this work now is to enlist co-operation from other groups in pursuing these differences, particularly when appropriate normal control data are available, and so that any available osmolal data can be recalculated as above. Age matching of compared groups may be important here and should perhaps be conducted at least on a linked percentiles basis. One comprehensive study of osmolal responses for groups of normal subjects, glaucoma suspects, and those with established chronic simple glaucoma was presented by Galin et al. (1963), and we have reworked these data to compare initial osmolality, peak drop, and osmolal sum response as above. The results are shown in Table 6. It seemed reasonable to assume that the glaucoma suspect group included some patients now definable as ocular hypertensives. However, the differences between groups were not significant in any direct respect, and we did not proceed to discriminant analysis. We regard this lack of difference between the ocular hypertension and chronic simple glaucoma groups as an inconclusive result, as the true categories of patients in the glaucoma suspect group are uncertain, age ranges are not stated, and these earlier data are unusual in some respects. Very little variation was found in the initial osmolality values between subjects and between groups, relationships between the ocular pressures and osmolar responses were not close (comment by Spaeth, 1967), and subjects in whom the peak osmolal response did not exceed 6 milliosmoles were excluded. This recalculation has none the less been an instructive exercise, and there is a lack of studies published in sufficient detail to allow intergroup comparisons as above, although the relevant data are no doubt variously held. We have also recalculated our own data after exclusion of subjects giving a very poor osmolar response (Table 1), and the group differences were not materially altered.

These differences in systemic water-load response profiles and in plasma potassium levels raise some interesting possibilities, including the value of discriminant analysis in the diagnosis of the individual case. The telling aspect arises when functions derived from retrospective data on patients with confirmed diagnoses are used in the assessment of new patients, and this is now to follow.

We are grateful to Dr Gillian Paterson and $\mathrm{Mr}$ Stephen Miller, of the Glaucoma Unit, Moorfields Eye Hospital, London, WC1, for their cooperation, to the patients and colleagues who took part in this study, to the Frost Trust for generous financial support, and above all to Professor Norman Ashton for his critical support-often pointed but always constructive-over the years.

\section{References}

Camptell, D. A., Gloster, J., and Tonks, E. L. (1955). Some observations on the water-drinking test in glaucomatous and non-glaucomatous subjects. British Journal of Ophthalmology, 39, 193-203.

Drance, S. M. (1958). Studies in haemodilution in relation to the water test in chronic simple glaucoma. Transactions of the Ophthalmological Societies of the United Kingdom, 78, 565-573.

El-Shewy, T. M. (1973). Pituitrin as an adjuvant to water drinking in chonic simple glaucoma. Experimental Eye Research, 17, 1-4.

Frankelson, E. N. (1974). The role of the water test in evaluation of glaucoma control. Canadian Journal of Ophthalmology, 9, 408-410.

Galin, M. A., Aizawa, F., and McLean, J. M. (1965). Haemodilution and intraocular pressure. Archives of Ophthalmology, 73, 25-31. 
Galin, M. A., Mestre, C., and Nano, H. (1963). The water provocative test. American Journal of Ophthalmology, 56, 554-561.

Iverson, D. G., and Brown, D. W. (1967). Diurnal variation of intraocular pressure and serum osmolarity. Experimental Eye Research, 6, 179-186.

Kronfeld, P. C. (1975). Water drinking and outflow facility. Investigative Ophthalmology, 14, 49-52.

Roth, J. A. (1974). Inadequate diagnostic value of the waterdrinking test. British Journal of Ophthalmology, 58, 55-61.

Spaeth, G. L. (1967). The water drinking test. Archives of Ophthalmology, 77, 50-58.
Spaeth, G. L., and Vacharat, N. H. (1972). Provocative tests and chronic simple glaucoma. British Journal of Ophthalmology, 56, 205-216.

Vucicevic, Z. M., Scheie H. G., Berry, A., Yaros, M., and Frauenhoffer, C. (1975). The importance and accuracy of the water drinking test and tonography. Annals of Ophthalmology, 7, 39-45.

Winder, A. F., Paterson, G., and Miller, S. J. H. (1974). Biochemical abnormalities associated with ocular hypertension and low tension glaucoma. Transactions of the Ophthalmological Societies of the United Kingdom, 94, 518-524. 